\title{
Relationship between illness duration, corpus callosum changes, and sustained attention dysfunction in major depressive disorder
}

\author{
Wenming Zhao ${ }^{1 \#}$, Daomin Zhu ${ }^{2,3,4 \#}$, Yu Zhang ${ }^{2,3,4}$, Cun Zhang ${ }^{1}$, Biao Zhang ${ }^{1}$, Ying Yang ${ }^{1}$, Jiajia Zhu ${ }^{1}$, \\ Yongqiang Yu ${ }^{1}$ \\ ${ }^{1}$ Department of Radiology, The First Affiliated Hospital of Anhui Medical University, Hefei, China; ${ }^{2}$ Department of Sleep Disorders, Affiliated \\ Psychological Hospital of Anhui Medical University, Hefei, China; ${ }^{3}$ Department of Sleep Disorders, Hefei Fourth People's Hospital, Hefei, China; \\ ${ }^{4}$ Anhui Mental Health Center, Hefei, China \\ \#These authors contributed equally to this work.
}

Correspondence to: Prof. Yongqiang Yu, MD; Jiajia Zhu, PhD, MD. Department of Radiology, The First Affiliated Hospital of Anhui Medical University, No. 218, Jixi Road, Shushan District, Hefei 230022, China. Email: cjr.yuyongqiang@vip.163.com; zhujiajiagraduate@163.com.

Background: Illness duration is the main index of cumulative illness severity during depression progression. Corpus callosum (CC) damage is among the most replicated neurobiological findings in major depressive disorder (MDD). We aimed to investigate the nature and extent of the association between illness duration and CC changes.

Methods: Ninety-six MDD patients and 50 controls underwent diffusion and resting-state functional magnetic resonance imaging (fMRI). White matter micro-structure and inter-hemispheric functional connectivity were quantified by fractional anisotropy (FA) and voxel-mirrored homotopic connectivity (VMHC). The CC was reconstructed by tractography and divided into five sub-regions. The associations of illness duration with FA of each CC sub-region and voxel-wise VMHC were examined using correlation analyses. Also, we investigated the potential relationship between illness duration, CC changes, and clinical variables using mediation analyses.

Results: In MDD patients, longer illness duration was selectively associated with lower FA of CC subregions 2 [partial correlation coefficient $(\mathrm{pr})=-0.269, \mathrm{P}=0.009$ ] and 5 (pr=-0.296, $\mathrm{P}=0.004)$ as well as higher VMHC in the supplementary motor areas ( $\mathrm{pr}=0.378, \mathrm{P}<0.001$ ), precuneus ( $\mathrm{pr}=0.384, \mathrm{P}<0.001$ ), and lingual gyrus ( $\mathrm{pr}=0.373, \mathrm{P}<0.001)$ connected by the affected $\mathrm{CC}$ sub-regions. Further subgroup analyses demonstrated pronounced FA decrease and VMHC increase in patients with illness duration over 20 years relative to healthy controls (HC) and other patient subgroups with shorter illness durations. Moreover, lower FA of CC sub-regions 2 and 5 mediated the association between longer illness duration and more severe sustained attention dysfunction.

Conclusions: These findings provide evidence for compromised structure yet compensatory function of the CC with increasing depression illness duration, which may inform effective antidepressant treatment strategies at different disease stages.

Keywords: Major depressive disorder (MDD); corpus callosum (CC); illness duration; white matter microstructure; inter-hemispheric functional connectivity; magnetic resonance imaging (MRI)

Submitted Aug 13, 2020. Accepted for publication Feb 14, 2021.

doi: 10.21037/qims-20-970

View this article at: http://dx.doi.org/10.21037/qims-20-970 


\section{Introduction}

Major depressive disorder (MDD) is a severe psychiatric disorder characterized by periods when individuals experience guilt, sadness, and a loss of interest or pleasure in their daily lives, which gives rise to a heavy social and economic burden (1). The clinical course of MDD exhibits chronicity with acute $v s$. remitted status and first episode $v s$. recurrence. Relapse rates reach up to $80 \%$ within 1 year of remission (2), and over $30 \%$ of patients with MDD still fail to achieve a complete remission (3). Therefore, a better understanding of the nature and extent of the relationship between illness progression and neurobiological changes in depression may inform effective therapeutic strategies at different disease stages. During depression progression, illness duration is usually thought to be the main index of cumulative illness severity (4) and has shown strong associations with various brain abnormalities (5-11).

The diffusion tensor imaging (DTI) technique enables the in vivo measurement of brain white matter microstructure (12). The most commonly used DTI measure is fractional anisotropy (FA), which estimates the degree to which tissue organization limits water molecules' diffusion, thus reflecting membrane and myelin profiles of white matter (13). DTI meta-analyses have revealed widespread white matter impairments captured by FA in patients with MDD $(5,14,15)$. Among abnormal white matter tracts, the corpus callosum (CC) appears to be consistently more affected, which is supported by prior empirical evidence for FA abnormalities in the genu (14-19), body $(5,14,15,17,18,20-23)$, and splenium $(17,21,23,24)$ of the CC in MDD. In light of these findings, we focused principally on the CC and drew attention to its association with illness duration in MDD. It is quite apparent that the CC plays a vital role in maintaining stable functional communication between hemispheres, i.e., inter-hemispheric homotopic functional connectivity is supported by the underlying structural connectivity of the CC (25). Therefore, we sought to make use of convergent and complementary measures of white matter micro-structure and homotopic functional connectivity derived from DTI and functional magnetic resonance imaging (fMRI) to assess the microstructure and function of the CC, respectively. Here, we used voxel-mirrored homotopic connectivity (VMHC) (26) to reflect inter-hemispheric functional connectivity because this approach has been widely applied to explore neural substrates underlying various mental disorders such as generalized anxiety disorder (27), seizures (28), MDD (29), and schizophrenia (30).

The CC is the largest white matter structure in the brain and interconnects the bilateral cerebral hemispheres to integrate motor, perceptual, high-level cognitive, and emotional regulatory functions $(31,32)$. The heterogeneous functions depend on the fact that the CC is a multifactorial construct that can be divided into different sub-regions based on the specific cortical regions that these sub-regions connect (33). Moreover, the brain structure-function relationship is rather complex, raising the concept that function is shaped and constrained by structure, while structure can be modified or compensated by function. Hence, investigating micro-structural and functional changes of the CC at the sub-regional level using a combination of DTI-based tractography and fMRI-based VMHC may further improve our understanding of the role of the CC in many brain disorders, such as generalized anxiety disorder (27), traumatic brain injury (34), and amyotrophic lateral sclerosis (35). However, there remains a dearth of studies of this kind in the current research realm of depression to our knowledge.

Our goals in this study were three-fold. First, we examined the associations of illness duration with FA of each CC sub-region in patients with MDD using DTIbased tractography. Second, we explored the associations of illness duration with inter-hemispheric homotopic functional connectivity using fMRI-based VMHC, and then further tested the relationship between structural and functional changes of the CC. Finally, we investigated the potential associations between illness duration, CC changes, and clinical variables using mediation analyses.

\section{Methods}

\section{Participants}

Patients were recruited consecutively from Department of Sleep Disorders, Affiliated Psychological Hospital of Anhui Medical University. We also enrolled healthy controls (HC) from the local community via poster advertisements. A total of 146 right-handed subjects were enrolled, including 96 patients with MDD and 50 gender- and age-matched HC. Following the International Classification of Diseases (ICD-10), two well-trained clinical psychiatrists confirmed the diagnoses of depression. We carefully screened HC to confirm an absence of any psychiatric illness. The exclusion criteria for all participants included (I) the presence of other psychiatric disorders such as substance-induced mood 
disorder, bipolar disorder, anxiety disorders, schizophrenia, substance abuse, or dependence; (II) a history of significant physical or neurological disease; (III) a history of head injury with loss of consciousness; (IV) contraindications for MRI such as pregnancy. Additional exclusion criterion for $\mathrm{HC}$ was a family history of major neurological or psychiatric illnesses among their first-degree relatives. We used the 24-item Hamilton Rating Scale for Depression (HAMD) (36) and the 14-item Hamilton Rating Scale for Anxiety (HAMA) (37) to assess the severity of depression and anxiety symptoms. Regular antidepressant medications were received by all patients with MDD, including selective serotonin reuptake inhibitors (SSRIs), serotonin-norepinephrine reuptake inhibitors (SNRIs), and noradrenergic and specific serotonergic antidepressants (NaSSA). This study was approved by the ethics committee of The First Affiliated Hospital of Anhui Medical University. Written informed consent was obtained from all participants after being given a complete description of the study.

\section{Cognition assessment}

A computerized version of the Continuous Performance Task-Identical Pairs (CPT-IP) (38) was used to measure sustained attention as there is evidence that deficit in sustained attention represents a typical feature of depression $(39,40)$. The stimuli were $2-, 3-$, or 4 -digit numbers in separate conditions, which yielded separate scores reflecting increasing memory load on digit span. Participants were asked to monitor numbers on a computer screen and respond to any consecutive presentation of identical stimuli by key pressing as quickly as possible. Responses to target trials (pairs that were identical and required a response) and catch trials (pairs that were similar but not identical) were scored as true and false positive responses. The main outcome variable of interest was d'-a well-established discrimination sensitivity index incorporating both true and false positive responses. CPT-IP-2, -3 , and -4 represented d' values corresponding to the number of digits.

\section{Image acquisition}

MRI data were acquired using a 3.0-Tesla MR system (Discovery MR750w, General Electric, Milwaukee, WI, USA) with a 24-channel head coil. During scanning, tight but comfortable foam and earplugs were used to minimize head movement and scanner noise. All subjects were instructed to relax, keep their eyes closed but not fall asleep, think of nothing in particular, and move as little as possible. All participants underwent a high-resolution three-dimensional T1-weighted brain volume (BRAVO) sequence with the following parameters: repetition time $(\mathrm{TR})=8.5 \mathrm{~ms}$; echo time $(\mathrm{TE})=3.2 \mathrm{~ms}$; inversion time $(\mathrm{TI})$ $=450 \mathrm{~ms}$; flip angle $(\mathrm{FA})=12^{\circ}$; field of view $(\mathrm{FOV})=256 \mathrm{~mm}$ $\times 256 \mathrm{~mm}$; matrix size $=256 \times 256$; slice thickness $=1 \mathrm{~mm}$, no gap; voxel size $=1 \mathrm{~mm} \times 1 \mathrm{~mm} \times 1 \mathrm{~mm} ; 188$ sagittal slices; and acquisition time $=296 \mathrm{~s}$. Resting-state blood-oxygenlevel-dependent (BOLD) fMRI data were acquired using a gradient-echo single-shot echo planar imaging (GRESS-EPI) sequence with the following parameters: TR $=2,000 \mathrm{~ms} ; \mathrm{TE}=30 \mathrm{~ms} ; \mathrm{FA}=90^{\circ} ; \mathrm{FOV}=220 \mathrm{~mm} \times 220 \mathrm{~mm}$; matrix size $=64 \times 64$; slice thickness $=3 \mathrm{~mm}$, slice gap $=1 \mathrm{~mm}$; 35 interleaved axial slices; 185 volumes; and acquisition time $=370 \mathrm{~s}$. DTI data were acquired by a spin-echo singleshot echo planar imaging (SE-SS-EPI) sequence with the following parameters: $\mathrm{TR}=10,000 \mathrm{~ms}$; $\mathrm{TE}=74 \mathrm{~ms}$; FA $=90^{\circ} ; \mathrm{FOV}=256 \mathrm{~mm} \times 256 \mathrm{~mm}$; matrix $=128 \times 128$; slice thickness $=3 \mathrm{~mm}$ without gap; 50 axial slices; 64 diffusion gradient directions $\left(b=1,000 \mathrm{~s} / \mathrm{mm}^{2}\right)$ plus five $b=0$ reference images; and acquisition time $=700 \mathrm{~s}$. Routine T2-weighted images were also collected to exclude any organic brain abnormality. None of the participants were excluded for visually inspected imaging artifacts.

\section{DTI data preprocessing and fiber tracking of the CC}

The DTI data were preprocessed using the software packages FMRIB Software Library (FSL, http://www. fmrib.ox.ac.uk/fsl) (41). First, all the diffusion volumes were aligned to the b0 images with an affine transformation to minimize image distortion by eddy currents and to reduce inter-volume head motion. Brain tissues were then extracted using FSL's Brain Extraction Tool (BET) (http://www. fmrib.ox.ac.uk/fsl/bet2). To facilitate manual definition of the CC sub-regions, DTI data were resampled into a $1 \mathrm{~mm} \times 1 \mathrm{~mm} \times 1 \mathrm{~mm}$ voxel. After the preprocessing steps, a Gaussian tensor model was fitted to each voxel using Diffusion Toolkit (DTK, http://trackvis.org/dtk), and an FA map was generated for each subject. The wholebrain fiber tractography was performed using the Fiber Assignment by Continuous Tracking (FACT) algorithm with the FA threshold of 0.2 , and the maximum curvature angle of $45^{\circ}$. The CC sub-regions were defined on the midsagittal section of the FA maps using TrackVis software (http://trackvis.org). Two trained raters who were blind to 

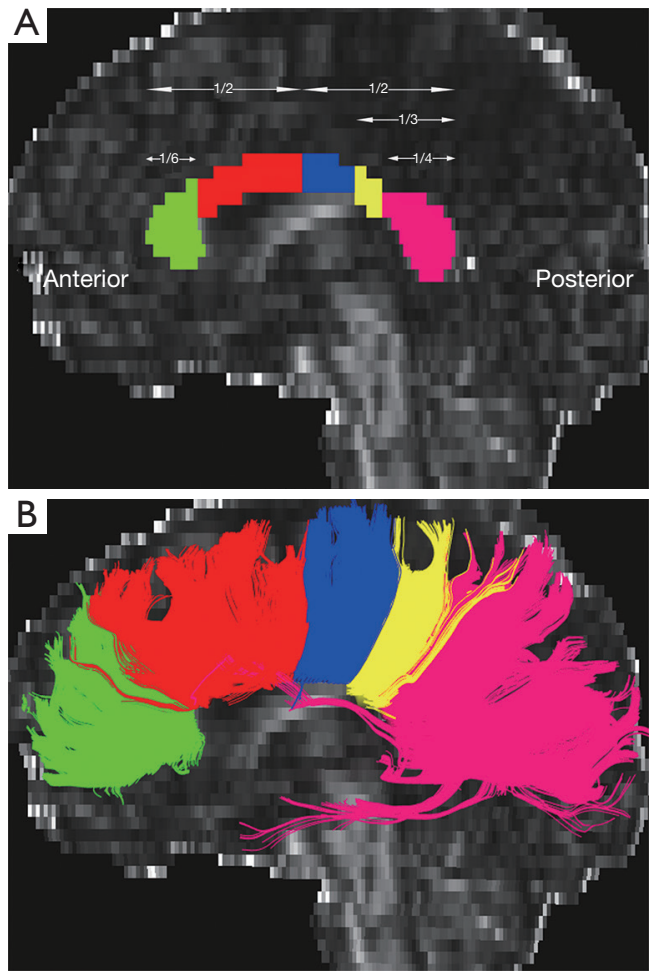

Figure 1 Sub-regions of the CC. Segmentation scheme of the CC (A). Fibers crossing through each sub-region (B). The most anterior sub-region 1 connects bilateral prefrontal cortices, subregion 2 premotor and supplementary motor cortices, sub-region 3 motor cortices, sub-region 4 sensory cortices, and the most posterior sub-region 5 parietal, temporal, and occipital cortices. CC, corpus callosum.

subjects' identity then manually segmented each CC into five sub-regions. The most anterior sub-region 1 connects bilateral prefrontal cortices, sub-region 2 premotor and supplementary motor cortices, sub-region 3 motor cortices, sub-region 4 sensory cortices, and the most posterior subregion 5 parietal, temporal, and occipital cortices (33) (Figure 1A). White matter fibers crossing through the five CC sub-regions were tracked separately (Figure 1B), and the average FA values of the five fibers were extracted for each subject. Inter-class correlation coefficients (ICC) ranged from 0.982 to 0.998 (Table S1) and Dice coefficients from 0.923 to 1 (Table S2), with the former representing the testretest reliability of inter-rater measurement and the latter reflecting the spatial similarity of inter-rater tracked fibers. Both measures suggested excellent inter-rater reliability. The mean values of the two raters' manual measurements were calculated for subsequent statistical analyses.

\section{fMRI data preprocessing and inter-hemispheric functional connectivity analysis}

Resting-state fMRI data were preprocessed using Statistical Parametric Mapping software (SPM12, http://www.fil.ion. ucl.ac.uk/spm) and Data Processing \& Analysis for Brain Imaging (DPABI, http://rfmri.org/dpabi) (42). The first 10 volumes for each subject were discarded, and the remaining volumes were corrected for the acquisition time delay between slices. Realignment was then performed to correct the motion between time points. Head motion parameters were computed by estimating the translation in each direction and the angular rotation on each axis for each volume. All data were within the defined motion thresholds (i.e., translational or rotational motion parameters less than $2.5 \mathrm{~mm}$ or $2.5^{\circ}$ ). We also calculated frame-wise displacement (FD), which indexes the volume-to-volume changes in head position. Several nuisance covariates (the estimated motion parameters based on the Friston-24 model, the linear drift, the white matter signal, the cerebrospinal fluid signal, and the spike volumes with FD $>0.5$ ) were regressed out from the data. Then, the datasets were band-pass filtered using a frequency range of 0.01 to $0.1 \mathrm{~Hz}$. In the normalization step, individual structural images were firstly co-registered with the mean functional image; the transformed structural images were then segmented and normalized to the Montreal Neurological Institute (MNI) space using a high-level nonlinear warping algorithm, i.e., the diffeomorphic anatomical registration through exponentiated Lie algebra DARTEL technique (43). Finally, each filtered functional volume was spatially normalized to MNI space using the deformation parameters estimated during the above step and resampled into a $3-\mathrm{mm}$ isotropic voxel.

Inter-hemispheric functional connectivity was measured by VMHC, which was computed using the DPABI software. To obtain a better correspondence between symmetric voxels, we transformed the functional images to a symmetric space. First, all normalized T1 images were averaged to create a mean T1 image, which was then averaged with its left-right mirrored version to generate a group-specific symmetrical template. Second, the nonlinear registration to standard space was refined for each subject using the symmetrical template and was then used to transform each subject's functional data to the symmetric space. All functional data sets were then smoothed with a $6 \mathrm{~mm}$ full-width at half-maximum (FWHM) Gaussian kernel. VMHC was defined as the functional connectivity 
between any pair of symmetric inter-hemispheric voxels, i.e., the Pearson's correlation coefficient between the time series of each voxel and that of its symmetrical inter-hemispheric counterpart (26). The resultant VMHC maps were Fisher Z-transformed to improve the normality.

\section{Statistical analysis}

The statistical analyses of demographic and clinical data were performed using the SPSS 23.0 software package (SPSS, Chicago, IL, USA). Two-sample $t$-tests were used to compare age, educational years, FD, HAMD, HAMA, and CPT-IP scores between patients with MDD and HC. Pearson chi-square test was used to test group differences in gender. A threshold of $\mathrm{P}<0.05$ was considered statistically significant (two-tailed).

We adopted a multi-stage approach to analyze brain imaging data. First, we tested for the associations between FA of each CC sub-region and illness duration using partial correlation analyses with age, gender, and educational years as covariates. Bonferroni correction was adopted to adjust significance levels for multiple comparisons $(\mathrm{P}<0.05 / 5=0.01)$. Second, several studies have demonstrated an effect of antidepressants on white matter changes in MDD (44-46). To exclude this effect, we considered antidepressant types (SSRIs, SNRIs, and NaSSA) as a categorical variable and then repeated the partial correlation analyses, additionally adjusting for antidepressant types. Third, we initially tested the associations between cerebral VMHC and illness duration in a voxel-wise manner using partial correlation analyses with age, gender, educational years, and FD as covariates.

Moreover, we also repeated the partial correlation analyses, additionally adjusting for antidepressant types. Then, we explored the relationship between structural and functional changes of the CC. That is, for significant clusters showing correlations with illness duration, we extracted the VMHC values and then investigated their associations with $\mathrm{FA}$ of the corresponding CC sub-regions connecting these regions. Fourth, for the CC sub-regions associated with illness duration, we further examined the relationships between their FA values and clinical variables (i.e., HAMD, HAMA, CPT-IP scores). A threshold of $\mathrm{P}<0.05$ was considered to indicate statistical significance.

Moreover, to explore whether the associations between illness duration and clinical variables were mediated by CC micro-structure in patients with MDD, mediation analyses were performed using the PROCESS macro (http://www. processmacro.org/) available for SPSS $(47,48)$, with illness duration, clinical variables, and FA values as independent, dependent, and mediating variables. Age, gender, and educational years were also considered covariates in the mediation analyses. Based on 5,000 bootstrap realizations, the significance of mediation effects was assessed by the bootstrap $95 \%$ confidence interval (CI) in the way a significant indirect effect is indicated when the bootstrap 95\% CI does not include zero. Finally, to explore the exact nature of the relationships between CC changes and illness duration, we divided the patients with MDD into six subgroups according to illness duration (49). We tested the differences in FA and VMHC in relation to illness duration between controls and patients as well as between patient subgroups.

\section{Results}

\section{Demographic and clinical features}

Demographic and clinical data of the sample are listed in Table 1. In brief, the patient and control groups did not differ in gender (chi-square test, $\chi^{2}=0.755, \mathrm{P}=0.385$ ), age (two-sample $t$-test, $\mathrm{t}=1.227, \mathrm{P}=0.222)$, and $\mathrm{FD}(\mathrm{t}=1.505$, $\mathrm{P}=0.206)$. However, patients with $\mathrm{MDD}$ had lower education levels $(\mathrm{t}=-4.152, \mathrm{P}<0.001)$, CPT-IP-2 $(\mathrm{t}=-2.015$, $\mathrm{P}=0.046)$, CPT-IP-3 $(\mathrm{t}=-2.965, \mathrm{P}=0.004)$ and CPTIP-4 $(t=-2.086, P=0.039)$, and higher HAMD $(t=19.480$, $\mathrm{P}<0.001)$ and HAMA $(\mathrm{t}=18.315, \mathrm{P}<0.001)$ than HC.

\section{CC structure and illness duration of depression}

In patients with MDD, we found significant negative correlations between illness duration and FA of CC subregion 2 [partial correlation coefficient $(\mathrm{pr})=-0.269$, $\mathrm{P}=0.009$ ] (Figure $2 A$ ) and sub-region $5(\mathrm{pr}=-0.296$, $\mathrm{P}=0.004)$ (Figure $2 B)(\mathrm{P}<0.05$, Bonferroni corrected). We also observed a nominally significant negative correlation between illness duration and FA of the entire CC ( $\mathrm{pr}=-0.264, \mathrm{P}=0.011)$. There were no significant correlations between illness duration and FA of the other CC sub-regions (Table S3). Moreover, after additionally adjusting for antidepressant types, the correlations between illness duration and FA of CC sub-region 2 and 5 remained significant (Table S4). For completeness, we also measured the area of each CC sub-region to reflect their macrostructural morphology (Appendix 1) and found significant negative correlations between illness duration and the area 
Table 1 Demographic and clinical characteristics of the sample

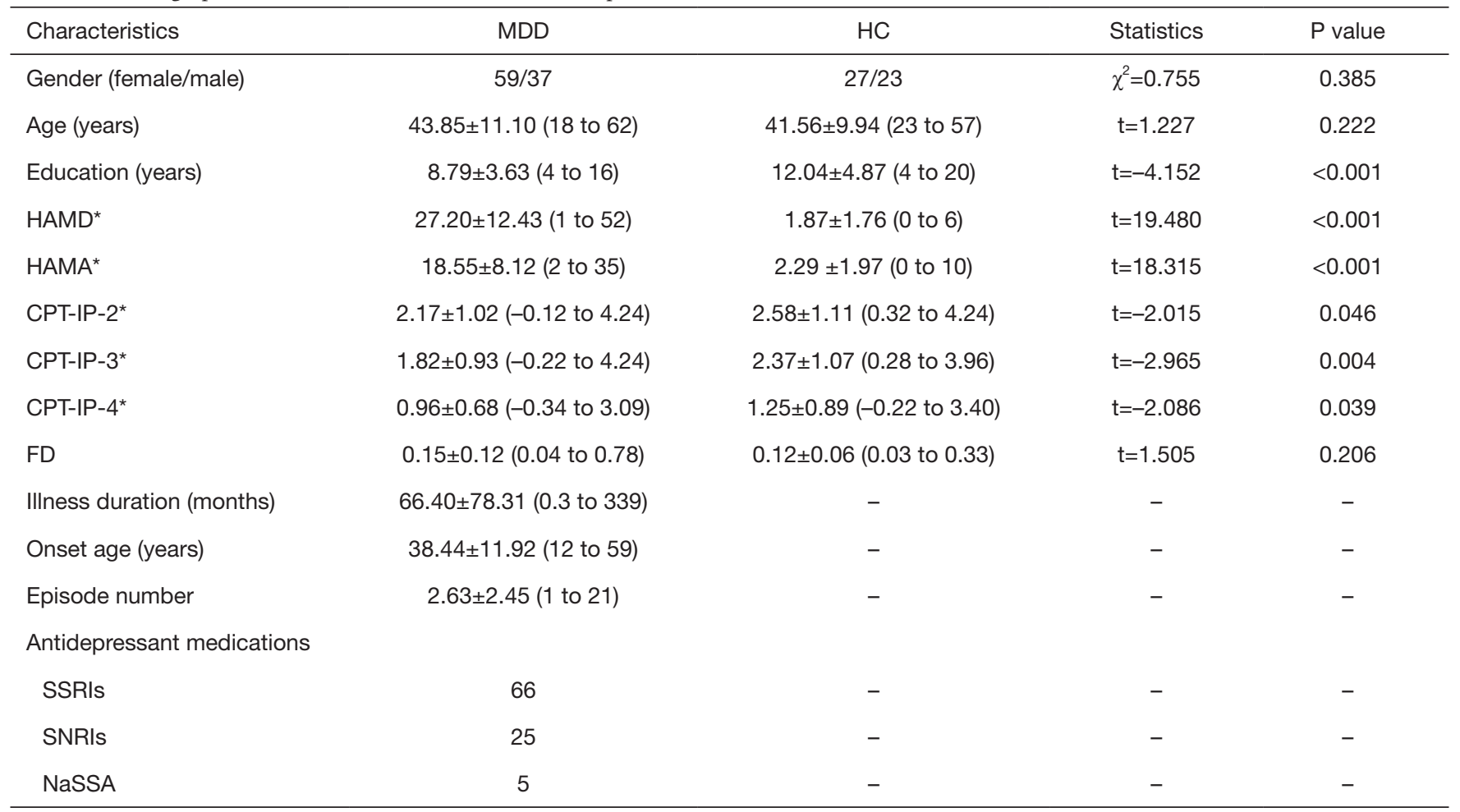

*, the data are available for 38 of $50 \mathrm{HC}$. Except for gender designation, data are means \pm SDs. Numbers in parentheses are the range. MDD, major depressive disorder; HC, healthy controls; HAMD, Hamilton Rating Scale for Depression; HAMA, Hamilton Rating Scale for Anxiety; CPT-IP, Continuous Performance Task-Identical Pairs; FD, frame-wise displacement; SSRIs, selective serotonin reuptake inhibitors; SNRIs, serotonin-norepinephrine reuptake inhibitors; NaSSA, noradrenergic and specific serotonergic antidepressant; SD, standard deviation.
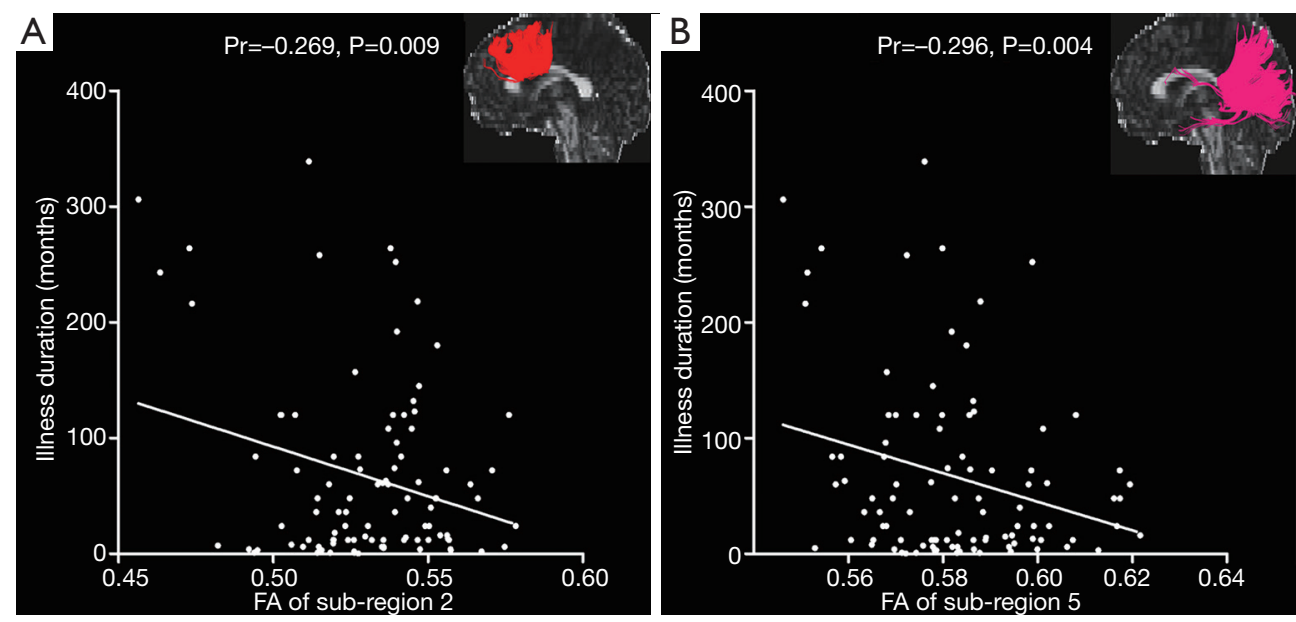

Figure 2 Correlations between illness duration and FA of the CC sub-regions. Scatter plots showing negative correlations between illness duration and FA of CC sub-region 2 (A) and sub-region 5 (B) in patients with MDD. FA, fractional anisotropy; CC, corpus callosum; MDD, major depressive disorder; pr, partial correlation coefficient. 


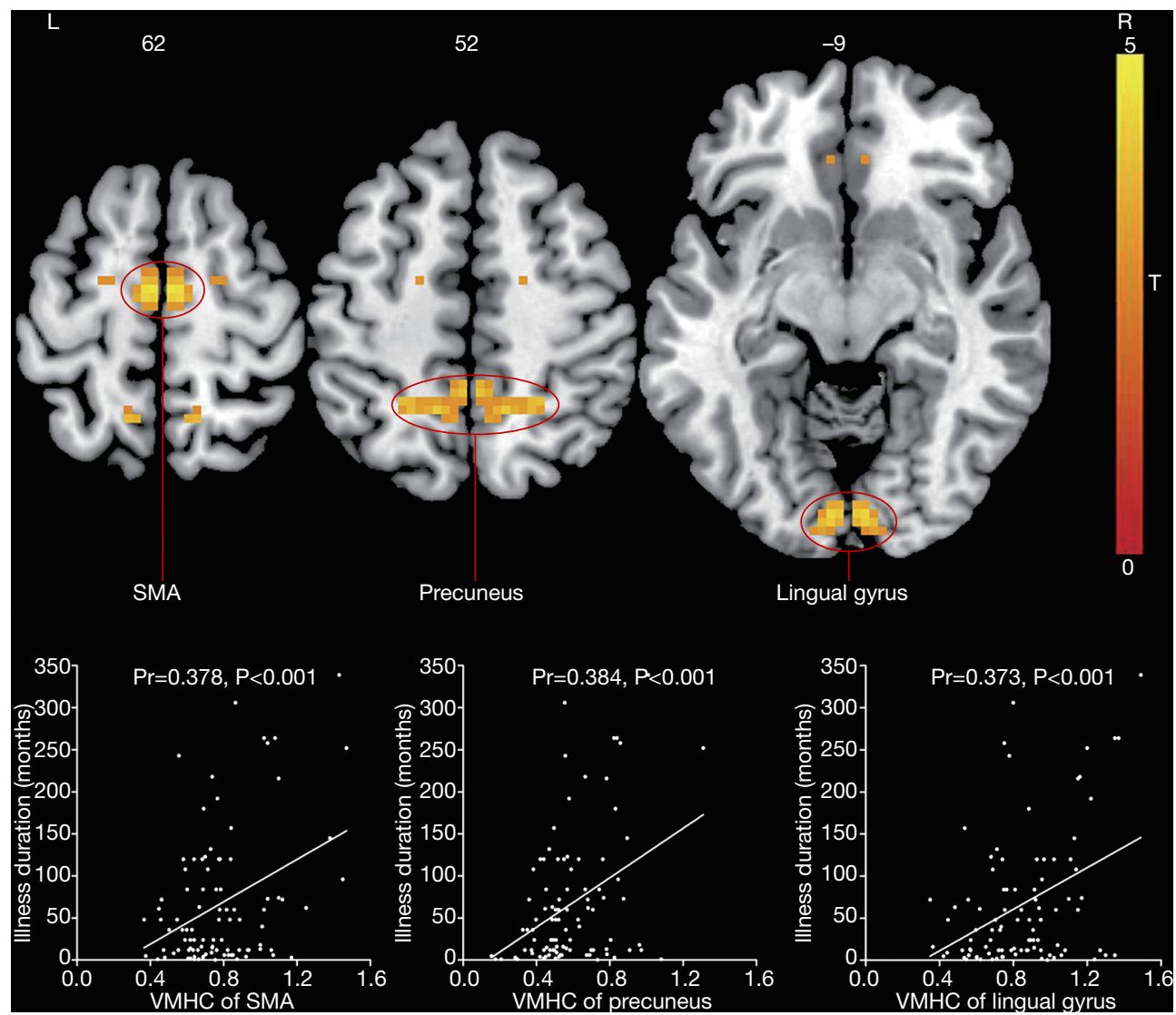

Figure 3 Brain regions with VMHC in relation to illness duration in patients with MDD. On the bottom, scatter plots show significant positive correlations between illness duration and VMHC of the significant clusters. VMHC, voxel-mirrored homotopic connectivity; MDD, major depressive disorder; L, left; R, right; SMA, supplementary motor area; pr, partial correlation coefficient.

of CC sub-region 3 ( $\mathrm{pr}=-0.287, \mathrm{P}=0.006)$ and sub-region 4 (pr=-0.285, $\mathrm{P}=0.006)$ (Figure S1 and Table S5).

\section{CC function and illness duration of depression}

Exploratory voxel-wise correlation analyses (uncorrected $\mathrm{P}<0.01$ and cluster size $>40$ voxels) revealed significant positive correlations between illness duration and VMHC in the supplementary motor area (peak coordinate: $\mathrm{x} / \mathrm{y} / \mathrm{z}=3$ / $-9 / 63 ; \mathrm{t}=3.908$; cluster size $=44$ voxels $)(\mathrm{pr}=0.378, \mathrm{P}<0.001)$, precuneus (peak coordinate: $\mathrm{x} / \mathrm{y} / \mathrm{z}=15 /-48 / 48 ; \mathrm{t}=3.873$; cluster size $=88$ voxels $)(\mathrm{pr}=0.384, \mathrm{P}<0.001)$, and lingual gyrus (peak coordinate: $\mathrm{x} / \mathrm{y} / \mathrm{z}=6 /-87 /-9 ; \mathrm{t}=3.735$; cluster size $=52$ voxels) $(\mathrm{pr}=0.373, \mathrm{P}<0.001)$ (Figure 3). Moreover, after additionally adjusting for antidepressant types, the correlations between illness duration and VMHC in the supplementary motor area, precuneus, and lingual gyrus remained significant (Table S4). However, no significant correlations were observed between FA of CC sub-region 2 and $\mathrm{VMHC}$ in the supplementary motor area (connected by sub-region 2) as well as between FA of CC sub-region 5 and VMHC in the precuneus and lingual gyrus (connected by sub-region 5) $(\mathrm{P}>0.05)$ (Table S6).

\section{Sensitivity analysis}

To test the possible effects of extreme outliers on our results, we repeated the correlation analyses between neuroimaging variables and illness duration after excluding participants with illness duration greater than mean +3 $x$ standard deviation $(\mathrm{SD})$ or smaller than mean $-3 \times \mathrm{SD}$. As shown in Table $\mathrm{S} 7$, despite changes in $\mathrm{P}$ values, most correlations remained nominally significant after excluding the outliers except the correlation between illness duration and FA of CC sub-region 2, indicating that the outliers influenced our results to an extent. 

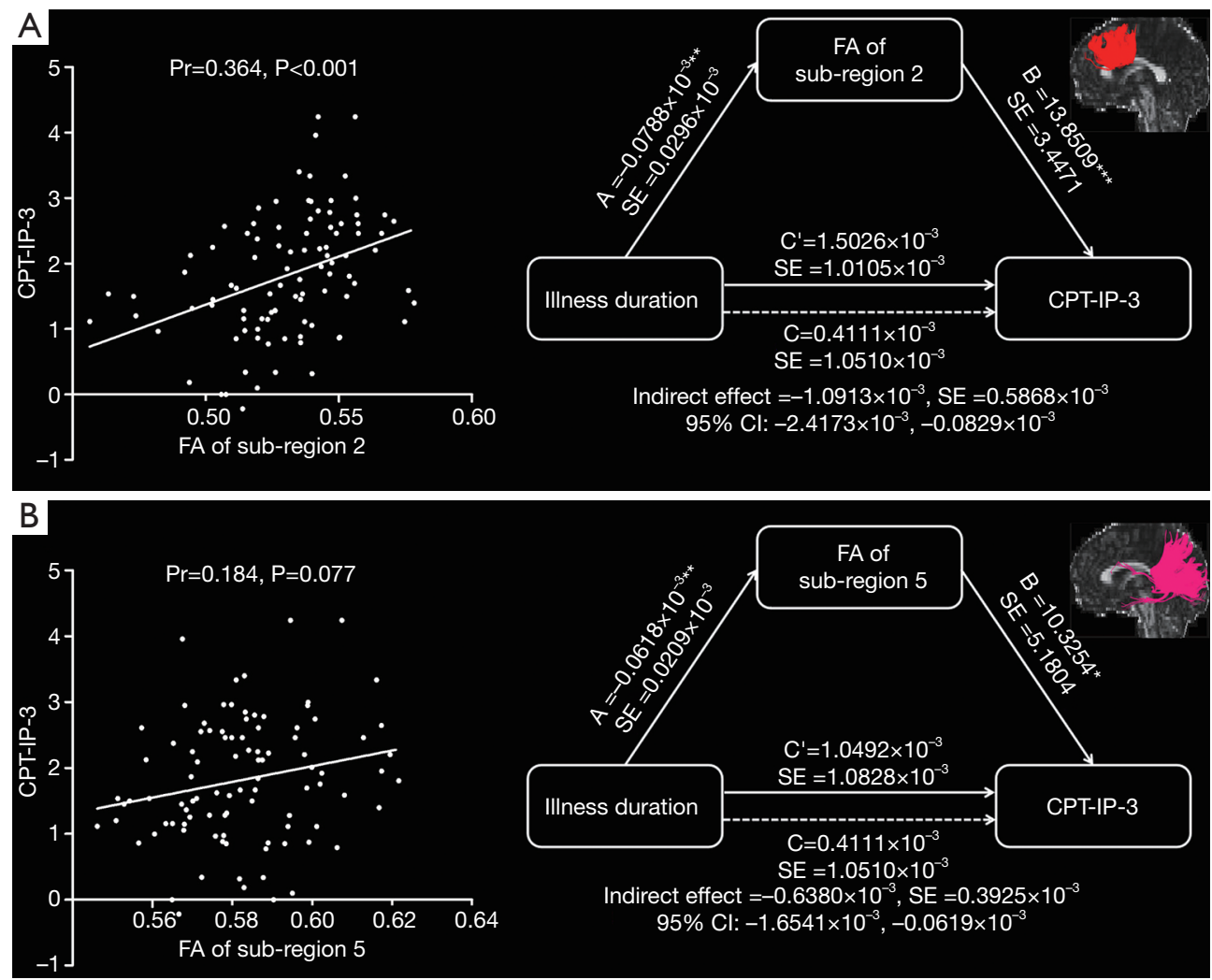

Figure 4 Associations between illness duration, FA of CC sub-region 2 (A) and sub-region 5 (B), and CPT-IP-3. On the left, scatter plots show positive correlations between illness duration and FA of sub-regions 2 and 5 . On the right, graphical representation of the mediation analyses between illness duration and CPT-IP-3, with FA of sub-regions 2 and 5 as the mediators: estimates of the mediated (a $\times$ b), direct (c'), and total (c) effects. All paths are reported as unstandardized ordinary least squares regression coefficients. ${ }^{*}, \mathrm{P}<0.05 ;{ }^{* *}, \mathrm{P}<0.01 ;{ }^{* * *}$, $\mathrm{P}<0.001$. FA, fractional anisotropy; CC, corpus callosum; CPT-IP, Continuous Performance Task-Identical Pairs; pr, partial correlation coefficient; SE, standard error.

\section{Associations between illness duration, CC micro-structure, and cognition}

In patients with MDD, we observed a significant positive correlation between CPT-IP-3 and FA of CC sub-region $2(\mathrm{pr}=0.364, \mathrm{P}<0.001)$ (Figure $4 A)$ and a trend towards a positive correlation between CPT-IP-3 and FA of CC subregion 5 ( $\mathrm{pr}=0.184, \mathrm{P}=0.077$ ) (Figure $4 B)$. No significant correlations were found between clinical symptoms (HAMD and HAMA) and FA of CC sub-regions 2 and 5 (Table S8).

In the mediation analysis model, all paths were reported as unstandardized ordinary least squares regression coefficients, namely, total effect of $\mathrm{X}$ on $\mathrm{Y}(\mathrm{c})=$ indirect effect of $\mathrm{X}$ on $\mathrm{Y}$ through $\mathrm{M}(\mathrm{a} \times \mathrm{b})+$ direct effect of $\mathrm{X}$ on $\mathrm{Y}\left(\mathrm{c}^{\prime}\right)$. In patients with MDD, we found that the relationship between illness duration and CPT-IP-3 was significantly mediated by FA of sub-region 2 (indirect effect
$=-1.0913 \times 10^{-3}, \mathrm{SE}=0.5868 \times 10^{-3}, 95 \%$ CI: $-2.4173 \times 10^{-3}$, $-0.0829 \times 10^{-3}$ ) (Figure $4 A$ ) and $\mathrm{FA}$ of sub-region 5 (indirect effect $=-0.6380 \times 10^{-3}, \mathrm{SE}=0.3925 \times 10^{-3}, 95 \% \mathrm{CI}$ : $\left.-1.6541 \times 10^{-3},-0.0619 \times 10^{-3}\right)$ (Figure 4B).

\section{Inter-group differences in structure and function of the CC}

There were no significant differences in FA of the entire $\mathrm{CC}$ and CC sub-regions or VMHC in the supplementary motor area, precuneus, and lingual gyrus between mixed patients and HC (Table S9). According to illness duration (49), we divided patients with MDD into six subgroups (subgroup $1, \leq 1$ year, $\mathrm{n}=33$; subgroup 2, $1-2$ years, $\mathrm{n}=12$; subgroup $3,2-5$ years, $\mathrm{n}=15$; subgroup $4,5-10$ years, $n=21$; subgroup $5,10-20$ years, $n=8$; and subgroup $6,>20$ years, $n=7$ ). Further pairwise comparisons 

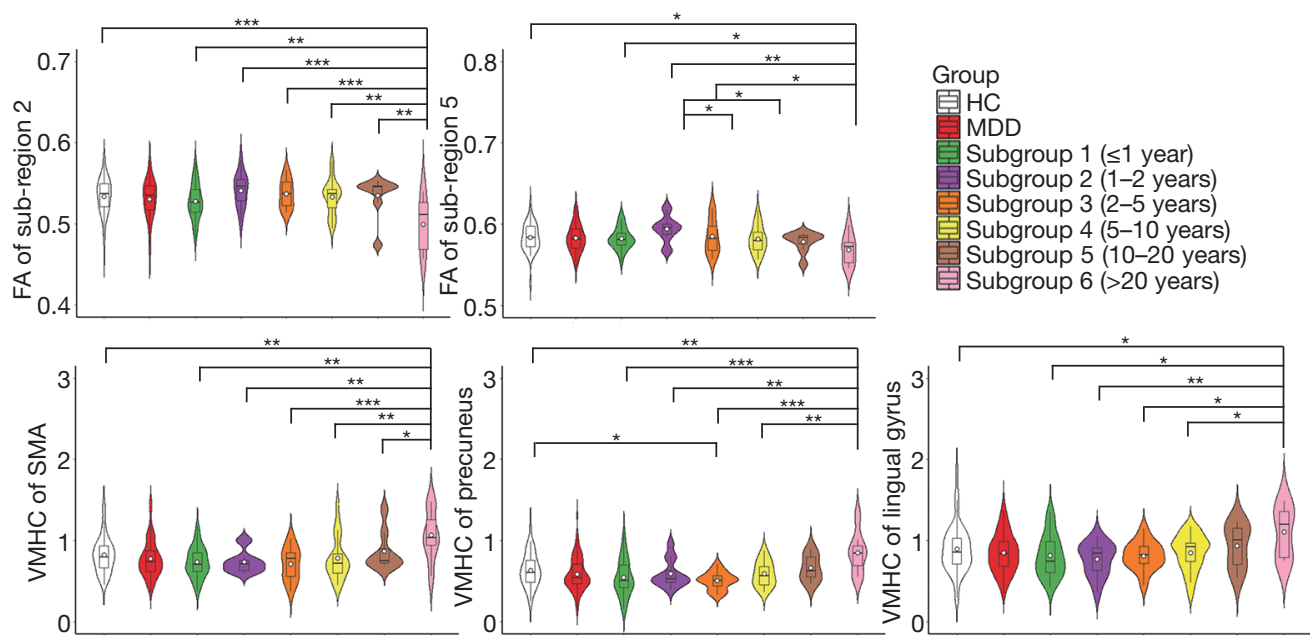

Figure 5 Inter-group differences in structure and function of the CC. *, $\mathrm{P}<0.05$; **, $\mathrm{P}<0.01$; ***, $\mathrm{P}<0.001$. CC, corpus callosum; FA, fractional anisotropy; VMHC, voxel-mirrored homotopic connectivity; SMA, supplementary motor area; HC, healthy controls; MDD, major depressive disorder.

revealed that subgroup 6 exhibited decreased FA of CC subregions 2 and 5 and increased VMHC in the supplementary motor area, precuneus, and lingual gyrus compared with $\mathrm{HC}$ and other patient subgroups with shorter illness durations (Figure 5).

\section{Discussion}

Using a combined analysis of DTI-based tractography and fMRI-based VMHC in a large cohort of patients with MDD, we found that longer illness duration (over 20 years in particular) was selectively associated with lower FA of CC sub-regions 2 and 5 as well as higher inter-hemispheric homotopic functional connectivity in the supplementary motor areas, precuneus, and lingual gyrus connected by the affected CC sub-regions. Also, lower FA of CC subregions 2 and 5 related to worse performance in sustained attention and acted as a mediator of the association between longer illness duration and more severe sustained attention dysfunction.

FA estimates the degree to which tissue organization limits water molecules' diffusion, which reflects regional white matter characteristics such as axon caliber, fiber density, and myelination (13). Changes in FA values could represent multiple underlying white matter micro-structural alterations, such as changes in axonal density or axonal diameter, abnormal myelination, or altered coherence of the fiber tracts (50). Here, we found significant negative correlations between illness duration and FA of CC sub- regions 2 and 5, suggesting that white matter microstructural features in the two sub-regions are preferentially affected by illness duration in MDD patients. The anterior sub-region 2 connects bilateral premotor and supplementary motor cortices, and the posterior sub-region 5 parietal, temporal, and occipital cortices (33). Interhemispheric information transfer between these homotopic cortical regions is vital for motor, emotional, and cognitive functions. Disturbed inter-hemispheric communications resulting from impaired white matter micro-structure of CC sub-regions 2 and 5 may affect these cortical regions and their functions, which is coherent with previous reports of structural and functional alterations in these regions in MDD (8,29,51,52). As a surrogate measure for cumulative illness severity during depression progression (4), illness duration has been linked to various brain impairments such as gray matter volume abnormalities $(6,7)$, functional connectivity changes $(8,9)$, and white matter microstructural alterations $(5,10,11)$. Our current observation that longer illness duration was predictive of greater white matter micro-structural disruption in the CC sub-regions may complement and extend previous literature on the relationship between illness duration and brain damage in depression. However, several DTI studies focusing principally on the CC failed to find a correlation between illness duration and white matter micro-structure of the CC $(21,53)$, which is inconsistent with our findings. This discrepancy may be explained by differences in sample sizes (96 vs. 18 and 20 patients), illness profiles (chronic 
vs. first-episode MDD), and methodology (fiber tracking $v s$. tract-based spatial statistics). Notably, the correlation between CC micro-structure and illness duration was still present after controlling for antidepressant types, indicating independence from medication.

Inter-hemispheric communication is an important organizational principle of the human brain, and the $\mathrm{CC}$ is mainly responsible for coordinating this communication (54). Previous neuroimaging studies have suggested that different cortical homotopic regions are anatomically connected by distinct CC sub-regions (55), with their inter-hemispheric functional connectivity facilitated by underlying CC structural connectivity (25). Given robust links between structural and functional connectivity in the human brain (56), it is natural to expect that decreased inter-hemispheric functional connectivity would be secondary to disrupted structural connectivity of the CC. Surprisingly, we found that longer illness duration correlated with higher VMHC in the supplementary motor areas, precuneus, and lingual gyrus connected by aberrant CC structural connectivity. The opposite findings regarding structural and functional changes may be explained by functionally compensatory processes for structural damage or concurrent structural and functional abnormalities. The former notion has been confirmed by a previous multi-modal MRI study reporting that enhanced inter-hemispheric functional connectivity between bilateral primary motor cortices compensates for their anatomical connection damages in subcortical stroke (57). However, we cannot rule out the possibility of concurrent structural hypoconnectivity and functional hyperconnectivity as no direct correlation between aberrant structural and functional connectivity was observed in the current study. There are at least two other reasons for this lack of association. First, the cortical homotopic regions connected by CC sub-regions 2 (premotor and supplementary motor cortices) and 5 (parietal, temporal, and occipital cortices) are much larger than the significant clusters identified in the voxel-wise VMHC correlation analyses, which may lead to a disproportional change pattern of the measured functional and structural connectivity. Second, although the CC serves as the major pathway for inter-hemispheric information transfer $(58,59)$, there is mounting evidence that other pathways contribute to inter-hemispheric synchronization, e.g., intact functional coupling between the hemispheres in individuals with complete agenesis of the CC (60) and early recovery of inter-hemispheric functional connectivity in epilepsy patients after total corpus callosotomy (61).
Behavioral evidence indicates that illness duration is a strong predictor of cognitive dysfunction in patients with MDD (62). Sustained attention abnormality is one of the typical cognitive deficits in depression $(39,40)$. Yamada et al. reported that micro-structural abnormalities in the CC segments connecting the frontal and parietal cortices were associated with working memory and attention impairments in patients with MDD (53), which is in agreement with the present findings of associations between lower CPTIP scores and lower FA in CC sub-regions 2 and 5. CC sub-regions 2 and 5 connect bilateral frontal and parietal cortices comprising the executive control network that is responsible for multiple cognitive functions, including sustained attention. Decreased FA in the two sub-regions may reflect disrupted inter-hemispheric communications between bilateral frontoparietal cortices, which may lead to dysfunction of the executive control network and subsequent deficit in sustained attention. Moreover, our further mediation analyses revealed that FA of CC subregions 2 and 5 mediated the relationship between illness duration and sustained attention, namely, more cumulative illness severity (indexed by longer illness duration) may cause greater CC micro-structural abnormity (indexed by lower FA), which may in turn result in worse performance in attention function. This finding may fill in the current gaps of knowledge regarding the exact relationship between illness duration and cognitive impairments in depression by identifying a mediating neural substrate.

This study did not find any group differences in micro-structure (FA) or function (VMHC) of the CC between mixed patients and HC. Case-control designs, which assume that patient and control groups are distinct entities, are overwhelmingly dominant in psychiatry but are limited to detecting group differences that essentially describe an average patient. Our further subgroup analyses demonstrated that patients with MDD with illness duration over 20 years exhibited pronounced FA decrease and interhemispheric functional connectivity increase relative to $\mathrm{HC}$ and other patient subgroups with shorter illness durations. This finding indicates that structural connectivity abnormity and functional connectivity compensation may occur in the later stage of depression and suggests that illness duration should be considered an important influence factor in depression research.

Several limitations should be considered when interpreting the present findings. First, all our patients were receiving antidepressant medication. Although findings were consistent when correcting for antidepressant types, 
the effect of medication cannot be completely eliminated, which may influence our interpretation. Future studies in medication-naive patients with MDD are needed to validate our preliminary findings. Second, the crosssectional and correlational design does not resolve causality. For example, we cannot exclude the possibility that more severe brain damage may render patients with MDD more prone to suffer from longer illness durations. Therefore, future longitudinal studies are required to establish the direction of causality. Finally, all the patients were recruited consecutively and randomly from the inpatient and outpatient departments. This contributes to the observation that most patients had a short illness duration, whereas the number of patients with a longer illness duration was rather small. In the future, more patients with a longer illness duration will be selectively enrolled to validate our preliminary results.

In summary, the present study revealed an association of increasing illness duration (over 20 years in particular) with compromised CC structural connectivity yet compensatory inter-hemispheric functional connectivity in MDD. Moreover, lower FA of the CC served as a mediator of the association between longer illness duration and more severe sustained attention dysfunction. These findings not only may help to clarify the relationship between illness duration, CC changes, and cognitive deficits in depression, but also may yield insights into effective antidepressant treatment strategies at different disease stages.

\section{Acknowledgments}

Funding: The study was supported by the National Natural Science Foundation of China (grant numbers: 81801679, 82071905, and 81771817), the Intercollegiate Key Projects of Nature Science of Anhui Province (grant number: KJ2018A0197), and the Anhui Natural Science Foundation (grant number: 201904a07020060).

\section{Footnote}

Conflicts of Interest: All authors have completed the ICMJE uniform disclosure form (available at http://dx.doi. org/10.21037/qims-20-970). The authors have no conflicts of interest to declare.

Ethical Statement: This study was approved by the ethics committee of The First Affiliated Hospital of Anhui Medical University. Written informed consent was obtained from all participants after they had been given a complete description of the study.

Open Access Statement: This is an Open Access article distributed in accordance with the Creative Commons Attribution-NonCommercial-NoDerivs 4.0 International License (CC BY-NC-ND 4.0), which permits the noncommercial replication and distribution of the article with the strict proviso that no changes or edits are made and the original work is properly cited (including links to both the formal publication through the relevant DOI and the license). See: https://creativecommons.org/licenses/by-nc-nd/4.0/.

\section{References}

1. GBD 2015 Disease and Injury Incidence and Prevalence Collaborators. Global, regional, and national incidence, prevalence, and years lived with disability for 310 diseases and injuries, 1990-2015: a systematic analysis for the Global Burden of Disease Study 2015. Lancet 2016;388:1545-602.

2. Fekadu A, Wooderson SC, Markopoulo K, Donaldson C, Papadopoulos A, Cleare AJ. What happens to patients with treatment-resistant depression? A systematic review of medium to long term outcome studies. J Affect Disord 2009;116:4-11.

3. Rush AJ, Trivedi MH, Wisniewski SR, Nierenberg AA, Stewart JW, Warden D, Niederehe G, Thase ME, Lavori PW, Lebowitz BD, McGrath PJ, Rosenbaum JF, Sackeim HA, Kupfer DJ, Luther J, Fava M. Acute and longerterm outcomes in depressed outpatients requiring one or several treatment steps: a STAR*D report. Am J Psychiatry 2006;163:1905-17.

4. Zaremba D, Enneking V, Meinert S, Forster K, Burger C, Dohm K, Grotegerd D, Redlich R, Dietsche B, Krug A, Kircher T, Kugel H, Heindel W, Baune BT, Arolt V, Dannlowski U. Effects of cumulative illness severity on hippocampal gray matter volume in major depression: a voxel-based morphometry study. Psychol Med 2018;48:2391-8.

5. Chen G, Guo Y, Zhu H, Kuang W, Bi F, Ai H, Gu Z, Huang X, Lui S, Gong Q. Intrinsic disruption of white matter microarchitecture in first-episode, drug-naive major depressive disorder: a voxel-based meta-analysis of diffusion tensor imaging. Prog Neuropsychopharmacol Biol Psychiatry 2017;76:179-87.

6. Serra-Blasco M, Portella MJ, Gomez-Anson B, de DiegoAdelino J, Vives-Gilabert Y, Puigdemont D, Granell E, 
Santos A, Alvarez E, Perez V. Effects of illness duration and treatment resistance on grey matter abnormalities in major depression. Br J Psychiatry 2013;202:434-40.

7. McKinnon MC, Yucel K, Nazarov A, MacQueen GM. A meta-analysis examining clinical predictors of hippocampal volume in patients with major depressive disorder. $\mathrm{J}$ Psychiatry Neurosci 2009;34:41-54.

8. Wang L, Li K, Zhang QE, Zeng YW, Jin Z, Dai WJ, Su YA, Wang G, Tan YL, Yu X, Si TM. Interhemispheric functional connectivity and its relationships with clinical characteristics in major depressive disorder: a resting state fMRI study. PLoS One 2013;8:e60191.

9. Fan H, Yang X, Zhang J, Chen Y, Li T, Ma X. Analysis of voxel-mirrored homotopic connectivity in medicationfree, current major depressive disorder. J Affect Disord 2018;240:171-6.

10. de Diego-Adeliño J, Pires P, Gómez-Ansón B, SerraBlasco M, Vives-Gilabert Y, Puigdemont D, MartínBlanco A, Alvarez E, Pérez V, Portella MJ. Microstructural white-matter abnormalities associated with treatment resistance, severity and duration of illness in major depression. Psychol Med 2014;44:1171-82.

11. Lai CH, $\mathrm{Wu}$ Y'T. Alterations in white matter microintegrity of the superior longitudinal fasciculus and anterior thalamic radiation of young adult patients with depression. Psychol Med 2014;44:2825-32.

12. Le Bihan D, Mangin JF, Poupon C, Clark CA, Pappata S, Molko N, Chabriat H. Diffusion tensor imaging: concepts and applications. J Magn Reson Imaging 2001;13:534-46.

13. Winston GP. The physical and biological basis of quantitative parameters derived from diffusion MRI. Quant Imaging Med Surg 2012;2:254-65.

14. Chen G, Hu X, Li L, Huang X, Lui S, Kuang W, Ai H, Bi F, Gu Z, Gong Q. Disorganization of white matter architecture in major depressive disorder: a meta-analysis of diffusion tensor imaging with tract-based spatial statistics. Sci Rep 2016;6:21825.

15. Jiang J, Zhao YJ, Hu XY, Du MY, Chen ZQ, Wu M, Li KM, Zhu HY, Kumar P, Gong QY. Microstructural brain abnormalities in medication-free patients with major depressive disorder: a systematic review and metaanalysis of diffusion tensor imaging. J Psychiatry Neurosci 2017;42:150-63.

16. Bessette KL, Nave AM, Caprihan A, Stevens MC. White matter abnormalities in adolescents with major depressive disorder. Brain Imaging Behav 2014;8:531-41.

17. Cole J, Chaddock CA, Farmer AE, Aitchison KJ, Simmons A, McGuffin P, Fu CH. White matter abnormalities and illness severity in major depressive disorder. Br J Psychiatry 2012;201:33-9.

18. Won E, Choi S, Kang J, Kim A, Han KM, Chang HS, Tae WS, Son KR, Joe SH, Lee MS, Ham BJ. Association between reduced white matter integrity in the corpus callosum and serotonin transporter gene DNA methylation in medication-naive patients with major depressive disorder. Transl Psychiatry 2016;6:e866.

19. Xu K, Jiang W, Ren L, Ouyang X, Jiang Y, Wu F, Kong L, Womer F, Liu Z, Blumberg HP, Tang Y, Wang F. Impaired interhemispheric connectivity in medication-naive patients with major depressive disorder. J Psychiatry Neurosci 2013;38:43-8.

20. Kieseppä T, Eerola M, Mäntylä R, Neuvonen T, Poutanen VP, Luoma K, Tuulio-Henriksson A, Jylhä P, Mantere O, Melartin T, Rytsälä H, Vuorilehto M, Isometsä E. Major depressive disorder and white matter abnormalities: a diffusion tensor imaging study with tract-based spatial statistics. J Affect Disord 2010;120:240-4.

21. Han KM, Choi S, Jung J, Na KS, Yoon HK, Lee MS, Ham BJ. Cortical thickness, cortical and subcortical volume, and white matter integrity in patients with their first episode of major depression. J Affect Disord 2014;155:42-8.

22. Guo WB, Liu F, Chen JD, Xu XJ, Wu RR, Ma CQ, Gao K, Tan CL, Sun XL, Xiao CQ, Chen HF, Zhao JP. Altered white matter integrity of forebrain in treatment-resistant depression: a diffusion tensor imaging study with tractbased spatial statistics. Prog Neuropsychopharmacol Biol Psychiatry 2012;38:201-6.

23. Xiao J, He Y, McWhinnie CM, Yao S. Altered white matter integrity in individuals with cognitive vulnerability to depression: a tract-based spatial statistics study. Sci Rep 2015;5:9738.

24. Korgaonkar MS, Grieve SM, Koslow SH, Gabrieli JD, Gordon E, Williams LM. Loss of white matter integrity in major depressive disorder: evidence using tract-based spatial statistical analysis of diffusion tensor imaging. Hum Brain Mapp 2011;32:2161-71.

25. Shen K, Misic B, Cipollini BN, Bezgin G, Buschkuehl M, Hutchison RM, Jaeggi SM, Kross E, Peltier SJ, Everling S, Jonides J, McIntosh AR, Berman MG. Stable longrange interhemispheric coordination is supported by direct anatomical projections. Proc Natl Acad Sci U S A 2015;112:6473-8.

26. Zuo XN, Kelly C, Di Martino A, Mennes M, Margulies DS, Bangaru S, Grzadzinski R, Evans AC, Zang YF, Castellanos FX, Milham MP. Growing together and growing apart: regional and sex differences in the lifespan 
developmental trajectories of functional homotopy. J Neurosci 2010;30:15034-43.

27. Wang W, Peng Z, Wang X, Wang P, Li Q, Wang G, Chen F, Chen X, Liu S. Disrupted interhemispheric resting-state functional connectivity and structural connectivity in firstepisode, treatment-naive generalized anxiety disorder. J Affect Disord 2019;251:280-6.

28. Ji GJ, Zhang Z, Xu Q, Zang YF, Liao W, Lu G. Generalized tonic-clonic seizures: aberrant interhemispheric functional and anatomical connectivity. Radiology 2014;271:839-47.

29. Hermesdorf M, Sundermann B, Feder S, Schwindt W, Minnerup J, Arolt V, Berger K, Pfleiderer B, Wersching H. Major depressive disorder: Findings of reduced homotopic connectivity and investigation of underlying structural mechanisms. Hum Brain Mapp 2016;37:1209-17.

30. Zhu J, Zhu DM, Qian Y, Li X, Yu Y. Altered spatial and temporal concordance among intrinsic brain activity measures in schizophrenia. J Psychiatr Res 2018;106:91-8.

31. Gazzaniga MS. Cerebral specialization and interhemispheric communication: does the corpus callosum enable the human condition? Brain 2000;123:1293-326.

32. Quigley M, Cordes D, Turski P, Moritz C, Haughton V, Seth R, Meyerand ME. Role of the corpus callosum in functional connectivity. AJNR Am J Neuroradiol 2003;24:208-12.

33. Hofer S, Frahm J. Topography of the human corpus callosum revisited--comprehensive fiber tractography using diffusion tensor magnetic resonance imaging. Neuroimage 2006;32:989-94.

34. Wang Z, Zhang M, Sun C, Wang S, Cao J, Wang KKW, Gan S, Huang W, Niu X, Zhu Y, Sun Y, Bai L. Single mild traumatic brain injury deteriorates progressive interhemispheric functional and structural connectivity. J Neurotrauma 2021;38:464-73.

35. Zhang J, Ji B, Hu J, Zhou C, Li L, Li Z, Huang X, Hu $\mathrm{X}$. Aberrant interhemispheric homotopic functional and structural connectivity in amyotrophic lateral sclerosis. J Neurol Neurosurg Psychiatry 2017;88:369-70.

36. Williams JB. A structured interview guide for the Hamilton Depression Rating Scale. Arch Gen Psychiatry 1988;45:742-7.

37. Thompson E. Hamilton Rating Scale for Anxiety (HAM-A). Occup Med (Lond) 2015;65:601.

38. Cornblatt BA, Risch NJ, Faris G, Friedman D, Erlenmeyer-Kimling L. The Continuous Performance Test, identical pairs version (CPT-IP): I. New findings about sustained attention in normal families. Psychiatry
Res 1988;26:223-38.

39. Han G, Klimes-Dougan B, Jepsen S, Ballard K, Nelson M, Houri A, Kumra S, Cullen K. Selective neurocognitive impairments in adolescents with major depressive disorder. J Adolesc 2012;35:11-20.

40. Rock PL, Roiser JP, Riedel WJ, Blackwell AD. Cognitive impairment in depression: a systematic review and metaanalysis. Psychol Med 2014;44:2029-40.

41. Smith SM, Jenkinson M, Woolrich MW, Beckmann CF, Behrens TE, Johansen-Berg H, Bannister PR, De Luca M, Drobnjak I, Flitney DE, Niazy RK, Saunders J, Vickers J, Zhang Y, De Stefano N, Brady JM, Matthews PM. Advances in functional and structural MR image analysis and implementation as FSL. Neuroimage 2004;23 Suppl 1:S208-19.

42. Yan CG, Wang XD, Zuo XN, Zang YF. DPABI: data processing \& analysis for (resting-state) brain imaging. Neuroinformatics 2016;14:339-51.

43. Ashburner J. A fast diffeomorphic image registration algorithm. Neuroimage 2007;38:95-113.

44. Taylor WD, Macfall JR, Boyd B, Payne ME, Sheline YI, Krishnan RR, Murali Doraiswamy P. One-year change in anterior cingulate cortex white matter microstructure: relationship with late-life depression outcomes. Am J Geriatr Psychiatry 2011;19:43-52.

45. Khalaf A, Edelman K, Tudorascu D, Andreescu C, Reynolds CF, Aizenstein H. White matter hyperintensity accumulation during treatment of late-life depression. Neuropsychopharmacology 2015;40:3027-35.

46. Zhang Y, Han Y, Wang Y, Zhang Y, Li L, Jin E, Deng L, Watts B, Golden T, Wu N. A MRS study of metabolic alterations in the frontal white matter of major depressive disorder patients with the treatment of SSRIs. BMC Psychiatry 2015;15:99.

47. Hayes AF. Beyond Baron and Kenny: Statistical Mediation Analysis in the New Millennium. Communication Monographs 2009;76:408-20.

48. Hayes AF. Introduction to mediation, moderation, and conditional process analysis: a regression-based approach. Journal of Educational Measurement 2014;51:335-7.

49. Hardeveld F, Spijker J, De Graaf R, Nolen WA, Beekman AT. Recurrence of major depressive disorder and its predictors in the general population: results from the Netherlands Mental Health Survey and Incidence Study (NEMESIS). Psychol Med 2013;43:39-48.

50. Sexton CE, Mackay CE, Ebmeier KP. A systematic review of diffusion tensor imaging studies in affective disorders. Biol Psychiatry 2009;66:814-23. 
51. Zhang H, Li L, Wu M, Chen Z, Hu X, Chen Y, Zhu $\mathrm{H}$, Jia $\mathrm{Z}$, Gong $\mathrm{Q}$. Brain gray matter alterations in first episodes of depression: a meta-analysis of whole-brain studies. Neurosci Biobehav Rev 2016;60:43-50.

52. Arnone D, Job D, Selvaraj S, Abe O, Amico F, Cheng Y, Colloby SJ, O'Brien JT, Frodl T, Gotlib IH, Ham BJ, Kim MJ, Koolschijn PC, Perico CA, Salvadore G, Thomas AJ, Van Tol MJ, van der Wee NJ, Veltman DJ, Wagner G, McIntosh AM. Computational meta-analysis of statistical parametric maps in major depression. Hum Brain Mapp 2016;37:1393-404.

53. Yamada S, Takahashi S, Ukai S, Tsuji T, Iwatani J, Tsuda K, Kita A, Sakamoto Y, Yamamoto M, Terada M, Shinosaki K. Microstructural abnormalities in anterior callosal fibers and their relationship with cognitive function in major depressive disorder and bipolar disorder: a tract-specific analysis study. J Affect Disord 2015;174:542-8.

54. Doron KW, Gazzaniga MS. Neuroimaging techniques offer new perspectives on callosal transfer and interhemispheric communication. Cortex 2008;44:1023-9.

55. Chao YP, Cho KH, Yeh CH, Chou KH, Chen JH, Lin CP. Probabilistic topography of human corpus callosum using cytoarchitectural parcellation and high angular resolution diffusion imaging tractography. Hum Brain Mapp 2009;30:3172-87.

56. Hermundstad AM, Bassett DS, Brown KS, Aminoff EM, Clewett D, Freeman S, Frithsen A, Johnson A, Tipper

Cite this article as: Zhao $\mathrm{W}$, Zhu D, Zhang Y, Zhang C, Zhang B, Yang Y, Zhu J, Yu Y. Relationship between illness duration, corpus callosum changes, and sustained attention dysfunction in major depressive disorder. Quant Imaging Med Surg 2021;11(7):2980-2993. doi: 10.21037/qims-20-970
CM, Miller MB, Grafton ST, Carlson JM. Structural foundations of resting-state and task-based functional connectivity in the human brain. Proc Natl Acad Sci U S A 2013;110:6169-74.

57. Liu J, Qin W, Zhang J, Zhang X, Yu C. Enhanced interhemispheric functional connectivity compensates for anatomical connection damages in subcortical stroke. Stroke 2015;46:1045-51.

58. van den Heuvel MP, Mandl RC, Kahn RS, Hulshoff Pol HE. Functionally linked resting-state networks reflect the underlying structural connectivity architecture of the human brain. Hum Brain Mapp 2009;30:3127-41.

59. Johnston JM, Vaishnavi SN, Smyth MD, Zhang D, He BJ, Zempel JM, Shimony JS, Snyder AZ, Raichle ME. Loss of resting interhemispheric functional connectivity after complete section of the corpus callosum. J Neurosci 2008;28:6453-8.

60. Tyszka JM, Kennedy DP, Adolphs R, Paul LK. Intact bilateral resting-state networks in the absence of the corpus callosum. J Neurosci 2011;31:15154-62.

61. Hung SC, Lee CC, Chen HH, Chen C, Wu HM, Lin CP, Peng SJ. Early recovery of interhemispheric functional connectivity after corpus callosotomy. Epilepsia 2019;60:1126-36.

62. Elgamal S, Denburg S, Marriott M, MacQueen G. Clinical factors that predict cognitive function in patients with major depression. Can J Psychiatry 2010;55:653-61. 


\section{Methods}

\section{Association between illness duration and area of the CC}

High-resolution structural images were used for area measurement of the CC. The mid-sagittal slice was selected to measure area of the $5 \mathrm{CC}$ sub-regions according to the Rosenberg et al. study (63). Measurements were also made by two raters, with ICC ranging from 0.929 to 0.986 . The mean values of the two raters' manual measurements were calculated for subsequent statistical analyses. In addition, total intracranial volume (TIV) was assessed by using the CAT12 toolbox (http://www.neuro. uni-jena.de/cat). Then, we tested for the associations between area of each CC sub-region and illness duration using partial correlation analyses with age, gender, educational years and TIV as covariates. Bonferroni correction was adopted to adjust significance levels for multiple comparisons $(\mathrm{P}<0.05 / 5=0.01)$.

\section{References}

63. Rosenberg DR, Keshavan MS, Dick EL, Bagwell WW, MacMaster FP, Birmaher B. Corpus callosal morphology in treatmentnaive pediatric obsessive compulsive disorder. Prog Neuropsychopharmacol Biol Psychiatry 1997;21:1269-83.
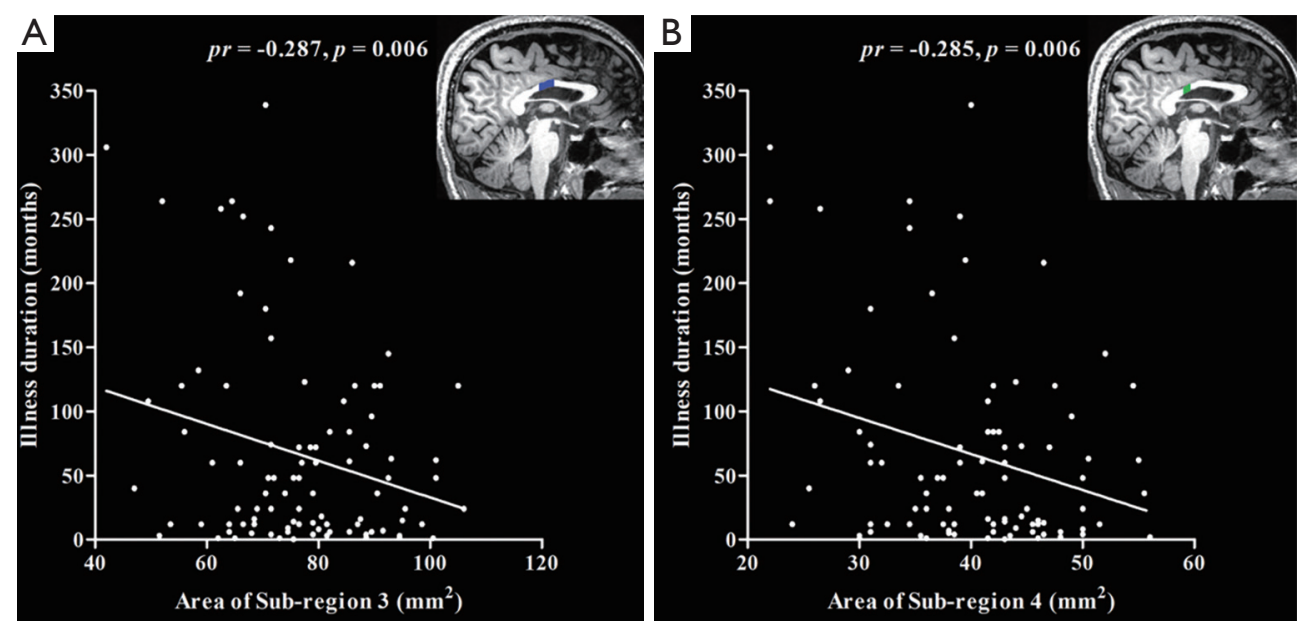

Figure S1 Scatter plots showing negative correlations between illness duration and area of CC sub-region 3 (A) and sub-region 4 (B) in patients with MDD. CC, corpus callosum; MDD, major depressive disorder; pr, partial correlation coefficient.

Table S1 Inter-rater reliability for FA values in the CC

\begin{tabular}{lcc}
\hline Region & ICC & P \\
\hline Sub-region 1 & 0.998 & $<0.001$ \\
Sub-region 2 & 0.994 & $<0.001$ \\
Sub-region 3 & 0.994 & $<0.001$ \\
Sub-region 4 & 0.994 & $<0.001$ \\
Sub-region 5 & 0.982 & $<0.001$ \\
\hline
\end{tabular}

FA, fractional anisotropy; CC, corpus callosum; ICC, inter-class correlation coefficients.

Table S3 Partial correlations between illness duration and FA of the CC sub-regions in patients with MDD

\begin{tabular}{lcc}
\hline \multirow{2}{*}{ Region } & \multicolumn{2}{c}{ Illness duration } \\
\cline { 2 - 3 } & $\operatorname{Pr}$ & $\mathrm{P}$ \\
\hline Sub-region 1 & -0.068 & 0.516 \\
Sub-region 2 & -0.269 & $0.009^{*}$ \\
Sub-region 3 & -0.196 & 0.060 \\
Sub-region 4 & -0.267 & 0.010 \\
Sub-region 5 & -0.296 & $0.004^{*}$ \\
\hline
\end{tabular}

*, $\mathrm{P}<0.05$, Bonferroni corrected. FA, fractional anisotropy; CC, corpus callosum; MDD, major depressive disorder; pr, partial correlation coefficient.
Table S2 Dice coefficients of inter-rater tracked fibers of the CC

\begin{tabular}{ll}
\hline Region & Range \\
\hline Sub-region 1 & $0.943-1$ \\
Sub-region 2 & $0.927-1$ \\
Sub-region 3 & $0.923-1$ \\
Sub-region 4 & $0.925-1$ \\
Sub-region 5 & $0.940-1$ \\
\hline
\end{tabular}

CC, corpus callosum.

Table S4 Partial correlations between illness duration and FA of the CC sub-regions as well as VMHC in the supplementary motor area, precuneus and lingual gyrus in patients with MDD after additionally adjusting for antidepressant types

\begin{tabular}{lcc}
\hline \multirow{2}{*}{ Imaging parameters } & \multicolumn{2}{c}{ Illness duration } \\
\cline { 2 - 3 } & $\mathrm{Pr}$ & $\mathrm{P}$ \\
\hline FA of sub-region 2 & -0.269 & 0.009 \\
FA of sub-region 5 & -0.295 & 0.005 \\
VMHC of supplementary motor area & 0.384 & $<0.001$ \\
VMHC of precuneus & 0.388 & $<0.001$ \\
VMHC of lingual gyrus & 0.371 & $<0.001$ \\
\hline
\end{tabular}

FA, fractional anisotropy; CC, corpus callosum; VMHC, voxel-mirrored homotopic connectivity; MDD, major depressive disorder; pr, partial correlation coefficient. 
Table S5 Partial correlations between illness duration and area of the CC sub-regions in patients with MDD, with age, gender, educational years and TIV as covariates

\begin{tabular}{lcc}
\hline \multirow{2}{*}{ Region } & \multicolumn{2}{c}{ Illness duration } \\
\cline { 2 - 3 } & $\operatorname{Pr}$ & $\mathrm{P}$ \\
\hline Sub-region 1 & -0.096 & 0.365 \\
Sub-region 2 & -0.244 & 0.019 \\
Sub-region 3 & -0.287 & $0.006^{\star}$ \\
Sub-region 4 & -0.285 & $0.006^{\star}$ \\
Sub-region 5 & -0.260 & 0.012 \\
\hline
\end{tabular}

*, $\mathrm{P}<0.05$, Bonferroni corrected. CC, corpus callosum; MDD, major depressive disorder; TIV, total intracranial volume; pr, partial correlation coefficient.

Table S6 Partial correlations between FA of CC sub-region 2 and VMHC in the supplementary motor area as well as between FA of CC subregion 5 and $\mathrm{VMHC}$ in the precuneus and lingual gyrus

\begin{tabular}{lcccc}
\hline \multirow{2}{*}{ VMHC } & \multicolumn{2}{c}{ FA of sub-region 2 } & \multicolumn{2}{c}{ FA of sub-region 5 } \\
\cline { 2 - 5 } \cline { 3 - 5 } & $\mathrm{Pr}$ & $\mathrm{P}$ & $\mathrm{Pr}$ & $\mathrm{P}$ \\
\hline Supplementary motor area & -0.131 & 0.210 & & 0.071 \\
Precuneus & & & -0.188 & 0.105 \\
Lingual gyrus & & -0.169 & \\
\hline
\end{tabular}

FA, fractional anisotropy; CC, corpus callosum; VMHC, voxel-mirrored homotopic connectivity; pr, partial correlation coefficient.

Table S7 Partial correlations between illness duration and FA of the CC sub-regions as well as VMHC in the supplementary motor area precuneus and lingual gyrus in patients with MDD after excluding the outliers

\begin{tabular}{lcc}
\hline \multirow{2}{*}{ Imaging parameters } & \multicolumn{2}{c}{ Illness duration } \\
\cline { 2 - 3 } & $\mathrm{Pr}$ & $\mathrm{P}$ \\
\hline FA of sub-region 2 & -0.161 & 0.127 \\
FA of sub-region 5 & -0.244 & 0.020 \\
VMHC of supplementary motor area & 0.321 & 0.002 \\
VMHC of precuneus & 0.373 & $<0.001$ \\
VMHC of lingual gyrus & 0.346 & 0.001 \\
\hline
\end{tabular}

FA, fractional anisotropy; CC, corpus callosum; VMHC, voxelmirrored homotopic connectivity; MDD, major depressive disorder; pr, partial correlation coefficient.

Table S8 Partial correlations between clinical symptoms and FA of CC sub-regions 2 and 5

\begin{tabular}{lcccccc}
\hline \multirow{2}{*}{ Clinical variables } & \multicolumn{2}{c}{ FA of sub-region 2 } & & \multicolumn{2}{c}{ FA of sub-region 5 } \\
\cline { 2 - 3 } \cline { 5 - 6 } & $\operatorname{Pr}$ & $\mathrm{P}$ & & $\operatorname{Pr}$ & $\mathrm{P}$ \\
\hline HAMD & 0.003 & 0.981 & & 0.017 & 0.873 \\
HAMA & -0.051 & 0.628 & & 0.032 & 0.764 \\
\hline
\end{tabular}

FA, fractional anisotropy; CC, corpus callosum; pr, partial correlation coefficient; HAMD, Hamilton Rating Scale for Depression; HAMA, Hamilton Rating Scale for Anxiety.
Table S9 Comparisons in FA of the entire CC and the CC subregions as well as in VMHC in the supplementary motor area, precuneus and lingual gyrus between mixed patients and HC

\begin{tabular}{lcc}
\hline Imaging parameters & $F$ & $P$ \\
\hline FA of the entire CC & 0.067 & 0.796 \\
FA of sub-region 1 & 1.125 & 0.291 \\
FA of sub-region 2 & 0.584 & 0.446 \\
FA of sub-region 3 & 0.240 & 0.625 \\
FA of sub-region 4 & 0.031 & 0.860 \\
FA of sub-region 5 & 0.308 & 0.580 \\
VMHC of supplementary motor area & 2.085 & 0.151 \\
VMHC of precuneus & 0.408 & 0.524 \\
VMHC of lingual gyrus & 0.823 & 0.366 \\
\hline
\end{tabular}

The FA values were compared between patients and controls by using a general linear model with age, gender and educational years as nuisance covariates. The VMHC values were compared between patients and controls by using a general linear mode with age, gender, educational years and FD as covariates. FA, fractional anisotropy; CC, corpus callosum; VMHC, voxelmirrored homotopic connectivity; HC, healthy controls; FD frame-wise displacement. 